\title{
Development of a high-throughput LC-APCI-MS method for the determination of 13 phytoestrogens (including gut microbial metabolites) in human urine and serum \\ C Wyns ${ }^{1}$, S Bolca ${ }^{1,2}$, D De Keukeleire ${ }^{1}$, A Heyerick ${ }^{1}$
}

1 Laboratory of Pharmacognosy and Phytochemistry, Faculty of Pharmaceutical Sciences, Harelbekestraat 72, Ghent University-UGent, B-9000 Ghent, Belgium

$\mathbf{2}$ Laboratory of Microbial Ecology and Technology, Faculty of Bioscience Engineering, Coupure Links 653, Ghent University-UGent, B-9000 Ghent, Belgium

Over the last few years the literature on the possible health benefits of phytoestrogens has expanded exponentially. However, potential use of phytoestrogens e.g. for the management of menopausal symptoms warrants further study in trials with rigorous scientific designs [1]. In view of the large interindividual variability in microbial metabolism of phytoestrogens in the large intestine, more consistent results for the use of phytoestrogens in a clinical environment can be expected, when there is a control for the absorption, metabolism and bioavailability. Large-scale studies require rapid, validated assays for the characterisation and quantification of the phytoestrogenic precursors and their metabolites in biological matrices. We report here a rapid, sensitive and specific HPLC-UV-atmospheric pressure chemical ionisation-mass spectrometry method for the simultaneous determination of 13 phytoestrogens (genistein, daidzein, equol, dihydrodaidzein, $O$-desmethylangolensin, coumestrol, secoisolariciresinol, matairesinol, enterodiol, enterolactone, isoxanthohumol, xanthohumol and 8-prenylnaringenin) in human urine and serum. The assay consists of a simple sample preparation procedure, using enzymatic deconjugation followed by liquid-liquid extraction (LLE) (urine) or solid phase extraction (SPE) (serum) and reversed-phase HPLC (XBridge ${ }^{\mathrm{TM}}, 3.5 \mu \mathrm{m}, 150 \mathrm{~mm} \times 3.0 \mathrm{~mm}$ i.d.) coupled to a photodiode array detector (DAD) and Multimode Source Detector (MSD) operating in APCI mode, using both positive and negative mode ionization. This method is suitable for the estimation of urinary and serum levels of the major phytoestrogens and their microbial metabolites (ppb-ppm range).

Acknowledgements: Research funded by a Ph.D grant of the Institute for the Promotion of Innovation through Science and Technology in Flanders (IWT-Vlaanderen) and was conducted in the framework of the FYTOES project (Agreement Nr. RT-05/02-FYTOES-1) sponsored by the Federal Public Service Health, Food Chain Safety and Environment.

References: 1. Nedrow, A. et al. (2006) Archives of internal medicine 166: 1453-1465. 